\title{
Examining the gastric cancer survival gap between Asians and whites in the United States
}

\author{
Hongbin Jin $^{1}$ (1) Paulo S. Pinheiro ${ }^{1} \cdot$ Karen E. Callahan $^{1} \cdot$ Sean F. Altekruse $^{2}$
}

Received: 21 April 2016/Accepted: 8 November 2016/Published online: 19 November 2016

(c) The International Gastric Cancer Association and The Japanese Gastric Cancer Association 2016

\begin{abstract}
Background Globally, Asian countries bear a disproportionate gastric cancer burden. Asian Americans, the fastest growing minority population in the US, have higher gastric cancer survival than non-Hispanic whites (NHWs) despite higher incidence. Benefitting from uniform cancer registry standards within the US, we examine for the first time the heterogeneity in the Asian American population, which may elucidate the causes of these disparities.

Methods SEER gastric cancer data from 2000 to 2012 were used to calculate 5-year survival estimates for NHWs and the six largest Asian ethnicities. Multivariate analyses were performed to identify critical prognostic factors and survival disparities between Asian groups and NHWs.

Results We analyzed 33,313 NHW and 8473 Asian gastric cancer cases. All Asian groups had significantly higher 5-year survival than NHWs, at 29.8\%. Among Asians, Koreans and Vietnamese had the highest and lowest survival, at $45.4 \%$ and $35.7 \%$, respectively. The Korean survival advantage was largely attributable to relatively high proportions of localized stage and low proportions of cardia tumors. After adjusting for major prognostic factors, the survival disadvantage of NHWs, while attenuated, remained significant in comparison to all Asian groups (HR: 1.33, 95\% CI: 1.24-1.43; reference: Korean). The
\end{abstract}

Hongbin Jin

hongbin.jin@unlv.edu

1 Department of Environmental and Occupational Health, University of Nevada, Las Vegas, 4505 S. Maryland Parkway, P.O. Box 453063, Las Vegas, NV 89154-3063, USA

2 Division of Cancer Control and Population Sciences, National Cancer Institute, Bethesda, MD, USA survival disparities within the Asian groups vanished with adjustment.

Conclusions This study characterizes distinctive gastric cancer survival patterns among the six major Asian groups and NHWs in the US. The favorable survival for Koreans is largely attributable to specific clinical factors, particularly stage at diagnosis. The causes of the survival disadvantage for NHWs remain elusive.

Keywords Gastric cancer - Survival · Disparities · Asian American · Epidemiology

\section{Introduction}

Gastric cancer is the third leading cause of cancer death and the fifth most common cancer worldwide, with some of the highest incidence and mortality rates found in the Eastern Asian countries of China, Japan, and Korea [1]. In the United States (US), the fastest growing minority population is Asian American, due to an immigration surge from these and other Asian countries, including India, Vietnam, and Philippines [2]. Not surprisingly, this ongoing demographic shift is impacting the gastric cancer profile in the US [1, 2]. While overall gastric cancer incidence and mortality rates have decreased steadily in the past two decades, survival remains relatively low compared to other cancers, at least in part due to a high proportion of diagnoses at an advanced stage [3].

Compared to non-Hispanic whites (NHWs), Asian Americans, as a whole, have higher gastric cancer incidence, but also have better survival outcomes [4-18]. Previous research, while not conclusive, has linked the survival advantage of Asian Americans to tumors at a more distal anatomic site, diagnosis at earlier tumor stages, 
diagnosis at younger ages, and more aggressive treatment approaches [6-18]. However, Asian Americans are heterogeneous, not only genetically, but also with respect to lifestyle, culture, immigration, and settlement experiences [19]. Aggregation of all Asians in epidemiological research ignores potential Asian subgroup variation in critical factors that impact cancer survival, including sociodemographic factors, tumor characteristics, healthcare access and quality, and cancer coping mechanisms [20]. Thus, we aim to identify and characterize differences in gastric cancer survival patterns between Asian subgroups in the US as well as between these subgroups and NHWs. Identifying the causes of possible survival disparities between Asians and NHWs has the potential to shed light on prognostic factors as well as protective attributes. The purpose of this study is to provide information to public health professionals tasked with reducing those disparities while improving cancer outcomes for all populations.

In the current study, we use the Surveillance, Epidemiology, and End Results (SEER) Program data from 2000 through 2012 to calculate 5-year gastric cancer survival estimates for NHWs and the six largest Asian subgroups in the US. Survival differences between these subgroups as well as in comparison to NHWs were examined after adjustment for the known important prognostic factors in gastric cancer survival.

\section{Materials and methods}

\section{Study population and covariates}

Population-based cancer data for NHWs and Asians (regardless of Hispanic ethnicity) aged 15 years or older were obtained from the SEER 18 registries, which cover $25 \%$ of the white and $50 \%$ of the Asian American population in the US [21]. Cases selected for analysis had an invasive tumor of the stomach diagnosed during the 13-year period from January 1, 2000 through December 31, 2012. Excluded cases were younger than 15 years old, diagnosed only at death or during autopsy, and those with a second or subsequent malignancy.

Net survival was calculated using a cause-specific survival framework, based on the SEER classification of cause-specific death [22]. Using the reported alive method, survival time was calculated in months from the date of diagnosis to whichever occurred first: the date of death from gastric cancer, the date of last alive follow-up, or the final date of the study period, December 31, 2012. Those with zero survival time were excluded; cases were censored at date lost to follow-up or date of death from other causes.
Eleven specific Asian subgroups are coded in the North American Association of Central Cancer Registries (NAACCR) standards: Asian Indian, Chinese, Filipino, Hmong, Japanese, Kampuchean, Korean, Laotian, Pakistani, Thai, and Vietnamese [23]. The NAACCR Asian/ Pacific Islander Identification Algorithm (NAPIIA) enhances the identification of Asian subgroup by using name and birthplace information [24]. We aggregated Asian Indian and Pakistani into one single category, South Asian, because the NAACCR protocol did not code them separately until 2010 [23], and examined the six largest US Asian subgroups, hereafter referred to as Asian ethnicities: Chinese, Filipino, Japanese, Korean, South Asian, and Vietnamese. Smaller Hmong, Kampuchean, Laotian, and Thai populations as well as Asian cases with unknown ethnicity were combined into a single Other Asian category; however, they were not included in the survival analyses.

Other sociodemographic variables assessed for impact on survival were sex, age, marital status, insurance status, and socioeconomic status (SES). International age standard survival classification categories were used to form five age groups: 15-44, 45-54, 55-64, 65-74, and $75+$ [25]. Insurance status was grouped into four mutually exclusive groups: insured, which included Medicare and private insurance; Medicaid, including dual-eligible Medicaid/Medicare cases; uninsured; and unknown. Data on SES, reflecting aspects of social stratification that play a critical role in cancer survival, are not routinely collected at the individual level by cancer registries. Using census tract information on cases, we adopted a quintile SES index that has been shown to detect socioeconomic gradients in cancer survival [26].

Routinely collected clinical data for each gastric cancer case, including primary anatomic site, histology, grade, and staging, were coded and reported according to the International Classification of Diseases for Oncology, Third Edition (ICD-O-3). Anatomic site was divided into four subsites: cardia (C16.0); middle, comprising the fundus, body, or curvatures (C16.1, C16.2, C16.5, and C16.6); distal, including the antrum or pylorus (C16.3 and C16.4); and overlapping or not otherwise specified (NOS) (C16.8 and C16.9). Histological types were categorized according to Lauren's classification and previous studies [27, 28] into diffuse type (codes 8020-8022, 8142, 8145, and 8490), intestinal type $(8140,8144,8210-8211,8260$, and 8480-8481), NOS (8000-8010), or other. Additional clinical covariates included SEER stage at diagnosis (localized, regional, distant, and unknown), tumor grade (I-IV and unknown), and treatment modality (surgery and radiation). 


\section{Statistical analyses}

Sociodemographic and clinical characteristics by race and Asian ethnicity were summarized with descriptive statistics. Five-year age-standardized overall survival, as well as survival stratified by anatomic site and stage at diagnosis, was calculated using the life table method [25].

Univariate analyses to determine significant prognostic factors were performed using the log-rank test, and covariates were tested for interaction effects. Multivariate survival analyses using Cox proportional hazards regression models produced hazard ratios (HRs) and corresponding 95\% confidence intervals (CIs) for risk of gastric cancer-specific mortality. The proportional hazards assumption, assessed by visual inspection of the $\log (-\log )$ plot of the survival distribution for each independent variable, had no significant violations. Variables were included via forward stepwise selection to assess the relative impact of significant prognostic factors. All statistical tests were two-sided with a significance level of 0.05 . All analyses were performed with SAS 9.3.

\section{Results}

A total of 33,313 NHW and 8473 Asian gastric cancer cases were studied. The distribution of Asians by ethnic group was as follows: $24 \%$ Korean, $24 \%$ Chinese, $21 \%$ Japanese, 12\% Filipino, 10\% Vietnamese, 5\% South Asian, and 5\% other Asian. Sociodemographic and clinical characteristics varied significantly between Asians and NHWs, as well as between Asian ethnicities (Table 1). In both races and every Asian ethnic group, cases were more likely to be male than female: the widest difference was seen in NHWs, at $64 \%$ male and $36 \%$ female; the narrowest in Filipinos, at $51 \%$ male and $49 \%$ female. Age at diagnosis distributions differed significantly, with a much higher proportion of South Asians (38\%) diagnosed younger than 55 years of age than NHWs (17\%) or any other Asian ethnicity. Conversely, Japanese cases had almost $80 \%$ of cases diagnosed at ages older than 65 , higher than all other comparison groups, including NHWs at $62 \%$ and Koreans at $56 \%$. The greatest variations were observed in gastric tumor characteristics. NHWs had a 3.5 times higher proportion of cardia tumors than Asians in the aggregate, but nearly 8 times higher than the largest Asian ethnic group in our study, Koreans. Most Asian ethnicities had a proportion similar to NHWs of tumors diagnosed at the localized stage, approximately $28 \%$, but Koreans had a larger share (35\%) and Vietnamese had much lower (23\%), resulting in a 1.5-fold difference between these two groups (Table 1).

Every Asian ethnic group had a significantly more favorable 5-year survival than NHWs, who had the lowest, at $29.8 \%$ (Table 2). Among Asians, Koreans had the highest survival at $45.4 \%$. Vietnamese and Filipinos were relatively low, at $35.7 \%$ and $36.4 \%$, respectively. After stratification by anatomic site, survival patterns in the Asian ethnic groups altered considerably, although NHWs retained significantly lower survival rates at every anatomic site. Chinese, South Asians, and Koreans showed the best survival for cardia, middle, and distal gastric cancer, respectively. Similarly, after stratification by stage at diagnosis, the survival advantage in Koreans only remained for localized gastric cancer, while Chinese and Filipinos had the highest survival in regional and distant gastric cancers, respectively. As with anatomic site, NHWs had worse survival than Asians for every stage of diagnosis.

In univariate survival analyses, the following variables were significant predictors for gastric cancer survival: sex, age at diagnosis, marital status, insurance status, SES, year of diagnosis, cancer registry, stage at diagnosis, anatomic site, histology, grade, treatment by surgery, and treatment by radiation. However, treatment modalities were not included in the multivariate survival analyses primarily because they were largely dependent upon stage at diagnosis and anatomic site, but also because cancer registry data do not differentiate between curative and palliative treatments. Due to strong interaction with SES and a high proportion of unknowns, insurance status was also not included. Similarly, tumor grade was excluded because of a significant interaction with stage at diagnosis.

Given the variations in 5-year survival by tumor characteristics, three separate models were generated to examine their impacts on racial and ethnic group disparities (Table 3). After adjusting for histology and other major prognostic variables (Model 1), Koreans showed significantly better survival than NHWs and every other Asian ethnic group. Adjusting for anatomic site yielded the same, although attenuated, results (Model 2). However, after taking into account stage at diagnosis, any survival disparity between Asian ethnicities disappeared (Model 3). Moreover, even after controlling for all prognostic factors available in our study, NHWs had a significant survival disadvantage compared to all Asians: $33 \%$ more likely to die after gastric cancer diagnosis.

In addition to race, other prognostic factors that significantly predicted gastric cancer survival were stage at diagnosis, which showed a 6.5 -fold increment in risk of death from distant stage to localized stage; histology, with diffuse type tumors predicting 1.23 times increased risk of death over intestinal type; and anatomic site, where cardia gastric tumors showed the worst survival, $16 \%$ increased risk over distal tumors. Additionally, the risk of death was $21 \%$ higher in the lowest SES quartile than the highest, and mortality risk steadily decreased with increasing SES. 
Table 1 Sociodemographic and clinical characteristics by race and Asian ethnicity in patients with gastric cancer, 2000-2012*

\begin{tabular}{|c|c|c|c|c|c|c|c|c|c|c|}
\hline \multirow[t]{3}{*}{ Characteristic } & \multirow{2}{*}{\multicolumn{2}{|c|}{$\frac{\text { Chinese }}{(N=2022)}$}} & \multirow{2}{*}{\multicolumn{2}{|c|}{$\frac{\text { Filipino }}{(N=990)}$}} & \multirow{2}{*}{\multicolumn{2}{|c|}{$\frac{\text { Japanese }}{(N=1739)}$}} & \multirow{2}{*}{\multicolumn{2}{|c|}{$\begin{array}{l}\text { Korean } \\
(N=2034)\end{array}$}} & \multirow{2}{*}{\multicolumn{2}{|c|}{$\frac{\text { South Asian }}{(N=416)}$}} \\
\hline & & & & & & & & & & \\
\hline & $N$ & $\%$ & $N$ & $\%$ & $N$ & $\%$ & $N$ & $\%$ & $N$ & $\%$ \\
\hline \multicolumn{11}{|l|}{ Sex } \\
\hline Male & 1153 & 57.0 & 506 & 51.1 & 932 & 53.6 & 1178 & 57.9 & 254 & 61.1 \\
\hline Female & 869 & 43.0 & 484 & 48.9 & 807 & 46.4 & 856 & 42.1 & 162 & 38.9 \\
\hline \multicolumn{11}{|l|}{ Age at diagnosis } \\
\hline $15-44$ & 142 & 7.0 & 74 & 7.5 & 37 & 2.1 & 154 & 7.6 & 75 & 18.0 \\
\hline $45-54$ & 223 & 11.0 & 121 & 12.2 & 116 & 6.7 & 300 & 14.7 & 82 & 19.7 \\
\hline $55-64$ & 341 & 16.9 & 214 & 21.6 & 215 & 12.4 & 439 & 21.6 & 95 & 22.8 \\
\hline $65-74$ & 502 & 24.8 & 245 & 24.7 & 426 & 24.5 & 625 & 30.7 & 85 & 20.4 \\
\hline $75+$ & 814 & 40.3 & 336 & 33.9 & 945 & 54.3 & 516 & 25.4 & 79 & 19.0 \\
\hline \multicolumn{11}{|l|}{ Insurance status } \\
\hline Uninsured & 50 & 2.5 & 28 & 2.8 & 12 & 0.7 & 106 & 5.2 & 29 & 7.0 \\
\hline Any Medicaid & 408 & 20.2 & 122 & 12.3 & 29 & 1.7 & 314 & 15.4 & 59 & 14.2 \\
\hline Insured & 1004 & 49.7 & 473 & 47.8 & 805 & 46.3 & 730 & 35.9 & 233 & 56.0 \\
\hline Unknown & 560 & 27.7 & 367 & 37.1 & 893 & 51.4 & 884 & 43.5 & 95 & 22.8 \\
\hline \multicolumn{11}{|l|}{ SES, quintile } \\
\hline 1 (lowest) & 250 & 12.4 & 103 & 10.4 & 115 & 6.6 & 323 & 15.9 & 32 & 7.7 \\
\hline 2 & 227 & 11.2 & 162 & 16.4 & 249 & 14.3 & 292 & 14.4 & 37 & 8.9 \\
\hline 3 & 318 & 15.7 & 229 & 23.1 & 376 & 21.6 & 291 & 14.3 & 54 & 13.0 \\
\hline 4 & 458 & 22.7 & 259 & 26.2 & 450 & 25.9 & 440 & 21.6 & 107 & 25.7 \\
\hline 5 & 739 & 36.5 & 229 & 23.1 & 536 & 30.8 & 610 & 30.0 & 180 & 43.3 \\
\hline Unknown & 30 & 1.5 & 8 & 0.8 & 13 & 0.7 & 78 & 3.8 & 6 & 1.4 \\
\hline \multicolumn{11}{|l|}{ Stage at diagnosis } \\
\hline Localized & 552 & 27.3 & 268 & 27.1 & 523 & 30.1 & 701 & 34.5 & 127 & 30.5 \\
\hline Regional & 684 & 33.8 & 289 & 29.2 & 544 & 31.3 & 655 & 32.2 & 110 & 26.4 \\
\hline Distant & 585 & 28.9 & 349 & 35.3 & 534 & 30.7 & 518 & 25.5 & 134 & 32.2 \\
\hline Unknown & 201 & 9.9 & 84 & 8.5 & 138 & 7.9 & 160 & 7.9 & 45 & 10.8 \\
\hline \multicolumn{11}{|l|}{ Anatomic site } \\
\hline Proximal/Cardia & 222 & 11.0 & 192 & 19.4 & 230 & 13.2 & 99 & 4.9 & 93 & 22.4 \\
\hline Middle & 601 & 29.7 & 299 & 30.2 & 567 & 32.6 & 684 & 33.6 & 121 & 29.1 \\
\hline Distal & 736 & 36.4 & 242 & 24.4 & 531 & 30.5 & 780 & 38.3 & 90 & 21.6 \\
\hline Overlapping/NOS & 463 & 22.9 & 257 & 26.0 & 411 & 23.6 & 471 & 23.2 & 112 & 26.9 \\
\hline \multicolumn{11}{|l|}{ Histology } \\
\hline Intestinal & 1226 & 60.6 & 523 & 52.8 & 1098 & 63.1 & 1263 & 62.1 & 198 & 47.6 \\
\hline Diffuse & 521 & 25.8 & 274 & 27.7 & 414 & 23.8 & 561 & 27.6 & 105 & 25.2 \\
\hline NOS & 84 & 4.2 & 32 & 3.2 & 52 & 3.0 & 78 & 3.8 & 9 & 2.2 \\
\hline Other & 191 & 9.4 & 161 & 16.3 & 175 & 10.1 & 132 & 6.5 & 104 & 25.0 \\
\hline \multirow[t]{3}{*}{ Characteristic } & \multicolumn{2}{|c|}{ Vietnamese } & \multicolumn{3}{|c|}{ Other Asian } & \multicolumn{2}{|c|}{ Total Asian } & \multicolumn{3}{|c|}{ NHW } \\
\hline & \multicolumn{2}{|c|}{$(N=866)$} & \multicolumn{3}{|c|}{$(N=406)$} & $(N=8$ & & $(N$ & 313) & \\
\hline & $N$ & $\%$ & & & $\%$ & $N$ & $\%$ & $N$ & & $\%$ \\
\hline Sex & & & & & & & & & & \\
\hline Male & 515 & 59.5 & & & 54.9 & 4761 & 56.2 & 21, & & 63.8 \\
\hline Female & 351 & 40.5 & & & 45.1 & 3712 & 43.8 & 12 & & 36.2 \\
\hline Age at diagnosis & & & & & & & & & & \\
\hline $15-44$ & 87 & 10.0 & & & 14.8 & 629 & 7.4 & & & 4.9 \\
\hline
\end{tabular}


Table 1 continued

\begin{tabular}{|c|c|c|c|c|c|c|c|c|}
\hline \multirow[t]{3}{*}{ Characteristic } & \multirow{2}{*}{\multicolumn{2}{|c|}{$\frac{\text { Vietnamese }}{(N=866)}$}} & \multirow{2}{*}{\multicolumn{2}{|c|}{$\begin{array}{l}\text { Other Asian } \\
(N=406)\end{array}$}} & \multirow{2}{*}{\multicolumn{2}{|c|}{$\frac{\text { Total Asian }}{(N=8473)}$}} & \multirow{2}{*}{\multicolumn{2}{|c|}{$\frac{\mathrm{NHW}}{(N=33,313)}$}} \\
\hline & & & & & & & & \\
\hline & $N$ & $\%$ & $N$ & $\%$ & $N$ & $\%$ & $N$ & $\%$ \\
\hline $45-54$ & 138 & 15.9 & 59 & 14.5 & 1039 & 12.3 & 3996 & 12.0 \\
\hline $55-64$ & 176 & 20.3 & 89 & 21.9 & 1569 & 18.5 & 7015 & 21.1 \\
\hline $65-74$ & 211 & 24.4 & 97 & 23.9 & 2191 & 25.9 & 8434 & 25.3 \\
\hline $75+$ & 254 & 29.3 & 101 & 24.9 & 3045 & 35.9 & 12,237 & 36.7 \\
\hline \multicolumn{9}{|l|}{ Insurance status } \\
\hline Uninsured & 20 & 2.3 & 14 & 3.4 & 259 & 3.1 & 959 & 2.9 \\
\hline Any Medicaid & 226 & 26.1 & 86 & 21.2 & 1244 & 14.7 & 1759 & 5.3 \\
\hline Insured & 320 & 37.0 & 168 & 41.4 & 3733 & 44.1 & 19,097 & 57.3 \\
\hline Unknown & 300 & 34.6 & 138 & 34.0 & 3237 & 38.2 & 11,498 & 34.5 \\
\hline \multicolumn{9}{|l|}{ SES, quintile } \\
\hline 1 (lowest) & 145 & 16.7 & 110 & 27.1 & 1078 & 12.7 & 4465 & 13.4 \\
\hline 2 & 189 & 21.8 & 68 & 16.7 & 1224 & 14.4 & 6290 & 18.9 \\
\hline 3 & 215 & 24.8 & 66 & 16.3 & 1549 & 18.3 & 7055 & 21.2 \\
\hline 4 & 171 & 19.7 & 70 & 17.2 & 1955 & 23.1 & 7545 & 22.6 \\
\hline 5 & 141 & 16.3 & 88 & 21.7 & 2523 & 29.8 & 7468 & 22.4 \\
\hline Unknown & 5 & 0.6 & 4 & 1.0 & 144 & 1.7 & 490 & 1.5 \\
\hline \multicolumn{9}{|l|}{ Stage at diagnosis } \\
\hline Localized & 197 & 22.7 & 114 & 28.1 & 2482 & 29.3 & 9429 & 28.3 \\
\hline Regional & 296 & 34.2 & 110 & 27.1 & 2688 & 31.7 & 8776 & 26.3 \\
\hline Distant & 305 & 35.2 & 133 & 32.8 & 2558 & 30.2 & 11,661 & 35.0 \\
\hline Unknown & 68 & 7.9 & 49 & 12.1 & 745 & 8.8 & 3447 & 10.3 \\
\hline \multicolumn{9}{|l|}{ Anatomic site } \\
\hline Proximal/cardia & 75 & 8.7 & 40 & 9.9 & 951 & 11.2 & 13,245 & 39.8 \\
\hline Middle & 255 & 29.4 & 115 & 28.3 & 2642 & 31.2 & 7468 & 22.4 \\
\hline Distal & 330 & 38.1 & 140 & 34.5 & 2849 & 33.6 & 5165 & 15.5 \\
\hline Overlapping/NOS & 206 & 23.8 & 111 & 27.3 & 2031 & 24.0 & 7435 & 22.3 \\
\hline \multicolumn{9}{|l|}{ Histology } \\
\hline Intestinal & 517 & 59.7 & 228 & 56.2 & 5053 & 59.6 & 20,814 & 62.5 \\
\hline Diffuse & 247 & 28.5 & 107 & 26.4 & 2229 & 26.3 & 6255 & 18.8 \\
\hline NOS & 29 & 3.3 & 20 & 4.9 & 304 & 3.6 & 1404 & 4.2 \\
\hline Other & 73 & 8.4 & 51 & 12.6 & 887 & 10.5 & 4840 & 14.5 \\
\hline
\end{tabular}

* Totals may not equal $100 \%$ due to rounding

\section{Discussion}

The striking difference in gastric cancer survival between Asian and Western countries has been the subject of much research [14-16, 29, 30]. With the burgeoning Asian American population in the US, SEER registries provide a unique platform to investigate this gap by examining differences not only between NHWs and Asians overall, but between specific Asian ethnic groups within the same country. The results of our study showed that all of the six largest Asian ethnicities in the US had significantly higher 5 -year survival than NHWs. Koreans had substantially higher 5-year survival than other Asian groups, especially
Vietnamese. However, the disparate gastric cancer survival between Asian subgroups could not be attributed to ethnicity alone; rather it more likely stems from a different case mix of important prognostic factors. Conversely, a persistent survival gap was observed between Asians and NHWs, even after adjustment for age, histology, subsite of the tumor, and other covariates. While sociodemographic factors such as younger age composition, better insurance, and higher SES improved prognosis for gastric cancer survival, tumor characteristics-notably, stage at diagnosis, histology, and anatomic site-were the most critical predictors, attenuating and/or eliminating observed ethnic and racial differences. 
Table 2 Age-standardized 5-year survival by race and Asian ethnicity in patients with gastric cancer, 2000-2012

\begin{tabular}{|c|c|c|c|c|c|c|c|c|c|c|}
\hline & \multicolumn{2}{|l|}{ Chinese } & \multicolumn{2}{|c|}{ Filipino } & \multicolumn{2}{|l|}{ Japanese } & \multicolumn{2}{|c|}{ Korean } & \multicolumn{2}{|c|}{ South Asian } \\
\hline & $\%$ & $(95 \% \mathrm{CI})$ & $\%$ & $(95 \% \mathrm{CI})$ & $\%$ & $(95 \% \mathrm{CI})$ & $\%$ & $(95 \% \mathrm{CI})$ & $\%$ & $(95 \% \mathrm{CI})$ \\
\hline \multicolumn{11}{|l|}{ Overall } \\
\hline & 42.2 & $(39.7-44.7)$ & 36.4 & $(32.8-40.0)$ & 38.6 & $(36.0-41.2)$ & 45.4 & $(43.0-47.9)$ & 43.4 & $(37.6-49.1)$ \\
\hline \multicolumn{11}{|l|}{ Anatomic site } \\
\hline Proximal/cardia & 37.1 & $(29.7-44.6)$ & 28.6 & $(20.5-36.7)$ & 28.4 & $(21.7-35.1)$ & 35.0 & $(23.7-46.3)$ & 25.1 & $(13.3-37.0)$ \\
\hline Middle & 51.5 & $(46.9-56.1)$ & 43.8 & $(37.1-50.4)$ & 49.8 & $(45.2-54.5)$ & 51.9 & $(47.6-56.1)$ & 54.7 & $(44.1-65.4)$ \\
\hline Distal & 44.2 & $(40.1-48.4)$ & 37.1 & $(29.9-44.3)$ & 43.0 & $(38.3-47.7)$ & 53.9 & $(50.0-57.9)$ & 46.8 & $(34.5-59.2)$ \\
\hline Overlapping/NOS & 28.8 & $(24.1-33.6)$ & 32.6 & $(25.9-39.3)$ & 27.2 & $(22.5-31.9)$ & 25.7 & $(21.1-30.3)$ & 40.8 & $(30.3-51.4)$ \\
\hline \multicolumn{11}{|l|}{ Stage } \\
\hline Localized & 77.7 & $(73.3-82.1)$ & 71.1 & $(64.3-77.9)$ & 77.6 & $(73.2-81.9)$ & 83.7 & $(80.5-86.9)$ & 76.8 & $(69.2-84.4)$ \\
\hline Regional & 44.7 & $(40.4-48.9)$ & 39.0 & $(31.9-46.0)$ & 41.7 & $(37.1-46.3)$ & 41.1 & $(36.9-45.4)$ & 36.7 & $(25.0-48.4)$ \\
\hline \multirow[t]{3}{*}{ Distant } & 9.0 & $(5.9-12.1)$ & 10.7 & $(6.9-14.6)$ & 7.7 & $(5.2-10.2)$ & 6.5 & $(3.9-9.2)$ & 7.2 & $(1.5-12.8)$ \\
\hline & \multicolumn{3}{|c|}{ Vietnamese } & \multicolumn{2}{|l|}{ Other Asian } & \multicolumn{3}{|c|}{ Total Asian } & \multicolumn{2}{|l|}{ NHW } \\
\hline & $\%$ & $(95 \%$ & & $\%$ & $(95 \% \mathrm{CI})$ & $\%$ & $(95$ & CI) & $\%$ & $(95 \% \mathrm{CI})$ \\
\hline \multicolumn{11}{|l|}{ Overall } \\
\hline & 35.7 & $(32.0-$ & & 36.8 & $(31.2-42.4)$ & 40.7 & $(39$ & 5-41.9) & 29.8 & $(29.2-30.3)$ \\
\hline \multicolumn{11}{|l|}{ Anatomic site } \\
\hline Proximal/cardia & 17.8 & $(6.5-$ & & 28.3 & $(10.3-46.3)$ & 30.9 & $(27$ & $3-34.4)$ & 23.1 & $(22.3-24.0)$ \\
\hline Middle & 47.3 & $(40.3-$ & 4) & 41.6 & $(30.5-52.7)$ & 49.4 & $(47$ & $2-51.6)$ & 41.4 & $(40.1-42.6)$ \\
\hline Distal & 39.7 & $(33.8$ & 7) & 33.0 & $(23.4-42.7)$ & 44.9 & $(42$ & $3-47.0)$ & 35.1 & $(33.7-36.6)$ \\
\hline Overlapping/NOS & 20.6 & $(14.1-$ & & 37.2 & $(27.6-46.7)$ & 28.4 & $(26$ & $-30.6)$ & 26.5 & $(25.3-27.6)$ \\
\hline \multicolumn{11}{|l|}{ Stage } \\
\hline Localized & 72.9 & $(65.3-$ & & 68.6 & $(58.5-78.8)$ & 78.3 & $(76$ & $3-80.3)$ & 66.1 & $(65.0-67.2)$ \\
\hline Regional & 41.2 & $(35.0$ & & 39.5 & $(28.5-50.5)$ & 40.7 & $(38$ & $5-42.9)$ & 27.0 & $(25.9-28.1)$ \\
\hline Distant & 9.2 & $(5.2-$ & .2) & 5.5 & $(0.0-11.0)$ & 7.8 & & $5-9.1)$ & 5.4 & $(4.9-5.9)$ \\
\hline
\end{tabular}

Because early diagnosis is critical to improving gastric cancer outcomes, there has been increased attention to the need for detecting tumors before symptoms are manifest [31-33]. For high-risk populations, research has shown that gastric cancer screening at a rational interval is cost-effective $[32,33]$. However, given the relatively low overall incidence and mortality rates in the US, population-based gastric cancer screening is not currently recommended. As such, the utilization of screening services largely depends on individuals' awareness of gastric cancer. In contrast, Japan and South Korea have implemented populationbased gastric cancer screening since 1983 and 1999, respectively, to increase early diagnosis and improve survival [34-36]. In our study, differences in stage at diagnosis distribution explain much of the Korean advantage in relation to other Asian ethnicities and to NHWs, as demonstrated by the model changes in Table 3. Koreans, the majority being first-generation immigrants $(79 \%$ foreign-born [20]), are more likely to be affected by the strong public awareness campaigns and accompanying national public health strategy for gastric cancer in South Korea.
Thus, the Korean communities in the US are apt to be proactive about gastric cancer screening, diagnosis, and treatment. On the other hand, since $68 \%$ of Japanese are US-born [20], their awareness of gastric cancer is likely

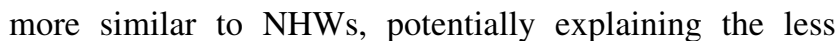
favorable stage distribution observed in this study, with proportions of localized tumors that are no different from that of NHWs.

Gastric cancer demonstrates marked heterogeneity at the histological level. Based on Lauren's classification, two major histologic subtypes, intestinal and diffuse type, are associated with different survival expectancy [27]. Concurrent with previous research, our study shows that cases with tumors of diffuse type have a significantly higher risk of death than those with the more common intestinal type [18, 28]. Diffuse type, more prevalent in females and young individuals, is characterized by the presence of poorly differentiated tumor cells [37, 38]. Given a higher male-to-female sex ratio and older age composition observed in NHWs, they had a lower proportion of diffuse type histology than Asians, as expected, but that contrasts 
Table 3 Risk of death from gastric cancer by prognostic factor among Asian American and non-Hispanic white patients, 2000-2012

\begin{tabular}{|c|c|c|c|c|c|c|c|c|c|}
\hline & \multicolumn{3}{|c|}{ Model $1^{*}$} & \multicolumn{3}{|c|}{ Model $2^{\dagger}$} & \multicolumn{3}{|c|}{ Model $3^{*}$} \\
\hline & HR & $(95 \% \mathrm{CI})$ & $p$ value & $\mathrm{HR}$ & $(95 \% \mathrm{CI})$ & $p$ value & HR & $(95 \% \mathrm{CI})$ & $p$ value \\
\hline \multicolumn{10}{|l|}{ Race/ethnicity } \\
\hline Korean & - & - & - & - & - & - & - & - & - \\
\hline Chinese & 1.15 & $(1.05-1.26)$ & $<0.01$ & 1.14 & $(1.04-1.24)$ & 0.01 & 1.01 & $(0.92-1.11)$ & 0.83 \\
\hline Japanese & 1.26 & $(1.15-1.39)$ & $<0.01$ & 1.23 & $(1.12-1.36)$ & $<0.01$ & 1.05 & $(0.95-1.16)$ & 0.32 \\
\hline Filipino & 1.38 & $(1.24-1.54)$ & $<0.01$ & 1.33 & $(1.19-1.48)$ & $<0.01$ & 1.10 & $(0.99-1.23)$ & 0.08 \\
\hline South Asian & 1.29 & $(1.10-1.52)$ & $<0.01$ & 1.24 & $(1.05-1.45)$ & 0.01 & 1.06 & $(0.90-1.24)$ & 0.48 \\
\hline Vietnamese & 1.27 & $(1.14-1.42)$ & $<0.01$ & 1.27 & $(1.14-1.42)$ & $<0.01$ & 1.07 & $(0.95-1.19)$ & 0.25 \\
\hline NHW & 1.70 & $(1.59-1.82)$ & $<0.01$ & 1.58 & $(1.48-1.70)$ & $<0.01$ & 1.33 & $(1.24-1.43)$ & $<0.01$ \\
\hline \multicolumn{10}{|l|}{ SES, quintile } \\
\hline 5 (highest) & - & - & - & - & - & - & - & - & - \\
\hline 4 & 1.06 & $(1.02-1.10)$ & $<0.01$ & 1.06 & $(1.02-1.10)$ & $<0.01$ & 1.05 & $(1.02-1.09)$ & 0.01 \\
\hline 3 & 1.10 & $(1.06-1.14)$ & $<0.01$ & 1.10 & $(1.06-1.14)$ & $<0.01$ & 1.12 & $(1.08-1.17)$ & $<0.01$ \\
\hline 2 & 1.13 & $(1.09-1.18)$ & $<0.01$ & 1.13 & (1.09-1.18) & $<0.01$ & 1.16 & $(1.11-1.20)$ & $<0.01$ \\
\hline 1 & 1.15 & $(1.10-1.21)$ & $<0.01$ & 1.16 & $(1.11-1.21)$ & $<0.01$ & 1.21 & $(1.15-1.26)$ & $<0.01$ \\
\hline Unknown & 1.02 & $(0.91-1.14)$ & 0.74 & 1.02 & $(0.91-1.14)$ & 0.74 & 1.05 & $(0.94-1.17)$ & 0.40 \\
\hline \multicolumn{10}{|l|}{ Histology } \\
\hline Intestinal & - & - & - & - & - & - & - & - & - \\
\hline Diffuse & 1.28 & $(1.24-1.32)$ & $<0.01$ & 1.28 & $(1.24-1.32)$ & $<0.01$ & 1.23 & $(1.19-1.27)$ & $<0.01$ \\
\hline NOS & 1.60 & $(1.51-1.69)$ & $<0.01$ & 1.41 & $(1.33-1.49)$ & $<0.01$ & 1.22 & $(1.15-1.30)$ & $<0.01$ \\
\hline Other & 0.32 & $(0.30-0.34)$ & $<0.01$ & 0.31 & $(0.30-0.33)$ & $<0.01$ & 0.41 & $(0.39-0.43)$ & $<0.01$ \\
\hline \multicolumn{10}{|l|}{ Anatomic site } \\
\hline Distal & & & & - & - & - & - & - & - \\
\hline Middle & & & & 1.05 & $(1.01-1.10)$ & 0.01 & 1.01 & $(0.97-1.06)$ & 0.54 \\
\hline Proximal/cardia & & & & 1.28 & $(1.23-1.33)$ & $<0.01$ & 1.16 & $(1.11-1.20)$ & $<0.01$ \\
\hline Overlapping/NOS & & & & 1.62 & $(1.56-1.69)$ & $<0.01$ & 1.30 & $(1.25-1.35)$ & $<0.01$ \\
\hline \multicolumn{10}{|l|}{ Stage at diagnosis } \\
\hline Localized & & & & & & & - & - & - \\
\hline Regional & & & & & & & 2.36 & $(2.26-2.46)$ & $<0.01$ \\
\hline Distant & & & & & & & 6.49 & $(6.23-6.76)$ & $<0.01$ \\
\hline Unknown & & & & & & & 3.37 & $(3.19-3.55)$ & $<0.01$ \\
\hline
\end{tabular}

* Model 1 was adjusted for race, sex, SES, age at diagnosis, marital status, year of diagnosis, cancer registry, and histology

${ }^{\dagger}$ Model 2 was adjusted for model 1 variables plus anatomic site

* Model 3 was adjusted for model 2 variables plus stage at diagnosis

with their survival disadvantage. In our study, the distribution of histological subtypes was similar across all Asian ethnicities; Filipinos and South Asians had a lower proportion of the favorable intestinal type histology, and Vietnamese had somewhat higher diffuse types.

Anatomic site determines treatment options and impacts gastric cancer survival. To date, surgery is the only curative treatment option, and the extent of gastric resection and margins largely depends on the location of the tumor. Tumors located in the distal part of the stomach are commonly treated by subtotal gastrectomy and reconstruction of digestive continuity. However, tumors located at the middle or proximal (cardia) part of the stomach may require total gastrectomy or esophagogastrectomy, if extended into the lower esophagus, resulting in a relatively worse prognosis [39-42]. Previous studies have shown that patients from Western countries have a significantly higher proportion of cardia tumors, while patients in Asia have a higher proportion of non-cardia gastric cancer. This variation could be attributed to risk factor prevalence in these different populations. A major risk factor for non-cardia gastric cancer is Helicobacter pylori infection; obesity and gastroesophageal reflux are associated with cancer in the cardia [43-46]. NHWs had a substantially higher proportion of cardia gastric cancer than Asians, yet even after stratification by anatomic demarcation, 5-year survival remained poor. Koreans had a remarkably low proportion of cardia gastric cancer, contributing further to their overall advantage. 
Asian race has been shown to be an independent prognostic factor for gastric cancer survival in many studies [6-17]. Here, we bolster those findings, demonstrating with multivariate analyses that each of the six major Asian ethnic groups has a survival advantage compared to NHWs. Critically, we found that the survival disparities between Asian ethnicities disappeared after controlling for major prognostic factors. To our knowledge, only one previous population-based study assessed the impact of specific Asian groups on gastric cancer survival. Kim et al., using Los Angeles County data, found significant survival disparities: Koreans had the highest and Filipinos had lowest gastric cancer survival [18]. Using the most current national data available, we found a significant gastric cancer survival disparity between NHWs and Asian Americans, but no significant differences within the Asian ethnic groups. In a separate analysis (data not shown), we analyzed receipt of surgery for localized stages, which are more likely to have curative intent, and found that NHWs had a lower proportion of surgery than Asians for each tumor anatomic site. However, in a survival model restricted to localized stage gastric cancer, differences in receipt of surgery were not enough to explain the disparities between Asians and NHWs. In short, the causes of the survival disadvantage for NHWs remain elusive; at the least, they are not discernible based on variables collected by SEER.

Several limitations may have affected our results. First, we used cause-specific death as our outcome, which may be impacted by cause of death misclassification on death certificates. Second, since Asians are more likely to have incomplete follow-up compared to NHWs and censoring across Asian ethnic groups is neither random nor even [47], it is possible that gastric cancer survival among Asians as a whole and/or by subgroup is overestimated. Loss to followup, which contributes to inflated survival estimates, may occur because of the return of immigrants with serious illnesses to their countries of origin to die, a phenomenon known as the salmon bias [47, 48]. However, studies thus far indicate that salmon bias has limited impact on Asian American survival, likely due to travel being too distant and time-consuming for gravely ill individuals to undertake [49, 50]. Lastly, some important covariates, such as residual tumor category, comorbidities, and postoperative complications, are critical factors impacting gastric cancer survival. However, we were unable to control for these confounding variables as such data are not routinely collected by US cancer registries.

This study characterizes the distinctive gastric cancer survival patterns among the six major Asian ethnic groups in the US and compares these patterns to NHWs. While there were observed survival differences between Asian ethnicities, these can largely be attributed to differences in major prognostic factors. In addition to the demographic and clinical characteristics studied here, gastric cancer awareness and coping mechanisms after diagnosis have important and lasting effects on cancer outcomes. Among immigrants, these are known to be associated with culture and length of stay in the US [51, 52]. Although the lack of survival disparities among Asian ethnicities does not explain the survival disadvantage of NHWs, revealed ethnic group differences point to the need for increased awareness among all Americans of gastric cancer screening as well as potential surgical options once diagnosed. With an increase in high-risk foreign-born Asian populations in the US reaching the ages of gastric cancer onset [2, 3], further public health efforts will be required to identify their protective survival attributes and prevent risk assimilation. Moreover, the vulnerability of NHWs for gastric cancer mortality has yet to be explained.

\section{Compliance with ethical standards}

Human rights statement and informed consent This article does not contain any studies with human or animal subjects performed by any of the authors.

Conflict of interest The authors declare that they have no conflict of interest.

\section{References}

1. International Agency for Research on Cancer. Stomach cancer. Estimated incidence, mortality and prevalence worldwide in 2012. http://globocan.iarc.fr/old/FactSheets/cancers/stomachnew.asp. Accessed 16 March 2016.

2. US Census Bureau. The Asian population: 2010. http://www. census.gov/prod/cen2010/briefs/c2010br-11.pdf. Accessed 16 March 2016.

3. Howlader N, Noone AM, Krapcho M, Garshell J, Miller D, Altekruse SF, et al. SEER cancer statistics review, 1975-2012. http://seer.cancer.gov/csr/1975_2012/. Accessed 16 March 2016.

4. Jin H, Pinheiro PS, Xu J, Amei A. Cancer incidence among Asian American populations in the United States, 2009-2011. Int J Cancer. 2016;. doi:10.1002/ijc.29958.

5. Gomez SL, Noone AM, Lichtensztajn DY, Scoppa S, Gibson JT, Liu L, et al. Cancer incidence trends among Asian American populations in the United States, 1990-2008. J Natl Cancer Inst. 2013;. doi:10.1093/jnci/djt157.

6. Wang J, Sun Y, Bertagnolli MM. Comparison of gastric cancer survival between Caucasian and Asian patients treated in the United States: results from the surveillance epidemiology and end results (SEER) database. Ann Surg Oncol. 2015;. doi:10.1245/ s10434-015-4388-4.

7. Bonenkamp JJ, van de Velde CJ, Kampschöer GH, Hermans J, Hermanek P, Bemelmans M, et al. Comparison of factors influencing the prognosis of Japanese, German, and Dutch gastric cancer patients. World J Surg. 1993;17:410-4.

8. Strong VE, Song KY, Park CH, Jacks LM, Gonen M, Shah M, et al. Comparison of gastric cancer survival following R0 resection in the United States and Korea using an internationally validated nomogram. Ann Surg. 2010;. doi:10.1097/SLA. 0b013e3181d3d29b. 
9. Davis PA, Sano T. The difference in gastric cancer between Japan, USA and Europe: what are the facts? What are the suggestions? Crit Rev Oncol Hematol. 2001;40:77-94.

10. Theuer CP, Kurosaki T, Ziogas A, Butler J, Anton-Culver H. Asian patients with gastric carcinoma in the United States exhibit unique clinical features and superior overall and cancer specific survival rates. Cancer. 2000;9:1883-92.

11. Nelson R, Ko EB, Arrington A, Lee W, Kim J, Garcia-Aguilar J, et al. Race and correlations between lymph node number and survival for patients with gastric cancer. J Gastrointest Surg. 2013; doi:10.1007/s11605-012-2125-x.

12. Howard JH, Hiles JM, Leung AM, Stern SL, Bilchik AJ. Race influences stage-specific survival in gastric cancer. Am Surg. 2015;81:259-67.

13. Theuer CP. Asian gastric cancer patients at a southern California comprehensive cancer center are diagnosed with less advanced disease and have superior stage-stratified survival. Am Surg. 2000;66:821-6.

14. Gill S, Shah A, Le N, Cook EF, Yoshida EM. Asian ethnicityrelated differences in gastric cancer presentation and outcome among patients treated at a Canadian cancer center. J Clin Oncol. 2003;21:2070-6.

15. Kim J, Sun CL, Mailey B, Prendergast C, Artinyan A, Bhatia S, et al. Race and ethnicity correlate with survival in patients with gastric adenocarcinoma. Ann Oncol. 2010;. doi:10.1093/annonc/ mdp290.

16. Merchant SJ, Li L, Kim J. Racial and ethnic disparities in gastric cancer outcomes: more important than surgical technique? World J Gastroenterol. 2014;. doi:10.3748/wjg.v20.i33.11546.

17. Schwarz RE, Zagala-Nevarez K. Ethnic survival differences after gastrectomy for gastric cancer are better explained by factors specific for disease location and individual patient comorbidity. Eur J Surg Oncol. 2002;28:214-9.

18. Kim J, Mailey B, Senthil M, Artinyan A, Sun CL, Bhatia S. Disparities in gastric cancer outcomes among Asian ethnicities in the USA. Ann Surg Oncol. 2009;. doi:10.1245/s10434-009-05844.

19. Pew Research Center. The rise of Asian Americans. http://www. pewsocialtrends.org/files/2013/04/Asian-Americans-new-fullreport-04-2013.pdf. Accessed 16 March 2016.

20. Gomez SL, Glaser SL, Horn-Ross PL, Cheng I, Quach T, Clarke CA, et al. Cancer research in Asian American, Native Hawaiian, and Pacific Islander populations: accelerating cancer knowledge by acknowledging and leveraging heterogeneity. Cancer Epidemiol Biomark Prev. 2014;. doi:10.1158/1055-9965.EPI-140624.

21. Surveillance, Epidemiology, and End Results Program. Number of persons by race and Hispanic ethnicity for SEER participants. http://seer.cancer.gov/registries/data.html. Accessed 16 March 2016.

22. Howlader N, Ries LA, Mariotto AB, Reichman ME, Ruhl J, Cronin KA. Improved estimates of cancer-specific survival rates from population-based data. J Natl Cancer Inst. 2010;. doi:10. 1093/jnci/djq366.

23. North American Association of Central Cancer Registries. NAACCR standards for cancer registries, volume II, data standards and data dictionary, version 16. http://www.naaccr.org/ Applications/ContentReader/Default.aspx. Accessed 16 March 2016.

24. Hsieh MC, Pareti LA, Chen VW. Using NAPIIA to improve the accuracy of Asian race codes in registry data. J Registry Manag. 2011;38:190-5

25. Corazziari I, Quinn M, Capocaccia R. Standard cancer patient population for age standardising survival ratios. Eur $\mathrm{J}$ Cancer. 2004;40:2307-16.
26. Yu M, Tatalovich Z, Gibson JT, Cronin KA. Using a composite index of socioeconomic status to investigate health disparities while protecting the confidentiality of cancer registry data. Cancer Causes Control. 2014;. doi:10.1007/s10552-013-0310-1.

27. Lauren $P$. The two histological main types of gastric carcinoma: diffuse and so-called intestinal-type carcinoma. An attempt at a histo-clinical classification. Acta Pathol Microbiol Scand. 1965;64:31-49.

28. Pinheiro PS, van der Heijden LH, Coebergh JW. Unchanged survival of gastric cancer in the southeastern Netherlands since 1982: result of differential trends in incidence according to Lauran type and subsite. Int J Cancer. 1999;84:28-32.

29. Bickenbach K, Strong VE. Comparisons of gastric cancer treatments: East vs West. J Gastric Cancer. 2012;. doi:10.5230/jgc. 2012.12.2.55.

30. Yamamoto M, Rashid OM, Wong J. Surgical management of gastric cancer: the East vs West perspective. J Gastrointest Oncol. 2015;. doi:10.3978/j.issn.2078-6891.2014.097.

31. Kim BJ, Heo C, Kim BK, Kim JY, Kim JG. Effectiveness of gastric cancer screening programs in South Korea: organized vs opportunistic models. World J Gastroenterol. 2013;. doi:10.3748/ wjg.v19.i5.736.

32. Dan YY, So JB, Yeoh KG. Endoscopic screening for gastric cancer. Clin Gastroenterol Hepatol. 2006;4:709-16.

33. Yeh JM, Hur C, Ward Z, Schrag D, Goldie SJ. Gastric adenocarcinoma screening and prevention in the era of new biomarker and endoscopic technologies: a cost-effectiveness analysis. Gut. 2016;. doi:10.1136/gutjnl-2014-308588.

34. Yoo KY. Cancer control activities in the Republic of Korea. Jpn J Clin Oncol. 2008;. doi:10.1093/jjco/hyn026.

35. Mizoue T, Yoshimura T, Tokui N, Hoshiyama Y, Yatsuya H, Sakata K, et al. Prospective study of screening for stomach cancer in Japan. Int J Cancer. 2003;106:103-7.

36. Hamashima C, Kim Y, Choi KS. Comparison of guidelines and management for gastric cancer screening between Korea and Japan. http://www.ispor.org/research_pdfs/49/pdffiles/PHS150. pdf. Accessed 16 March 2016.

37. Hu B, El Hajj N, Sittler S, Lammert N, Barnes R, Meloni-Ehrig A. Gastric cancer: classification, histology and application of molecular pathology. J Gastrointest Oncol. 2012;. doi:10.3978/j. issn.2078-6891.2012.021.

38. Yang D, Hendifar A, Lenz C, Togawa K, Lenz F, Lurje G, et al. Survival of metastatic gastric cancer: significance of age, sex and race/ethnicity. J Gastrointest Oncol. 2011;. doi:10.3978/j.issn. 2078-6891.2010.025.

39. Dikken JL, van de Velde CJ, Coit DG, Shah MA, Verheij M, Cats A. Treatment of resectable gastric cancer. Therap Adv Gastroenterol. 2012;. doi:10.1177/1756283X11410771.

40. Orditura M, Galizia G, Sforza V, Gambardella V, Fabozzi A, Laterza MM, et al. Treatment of gastric cancer. World J Gastroenterol. 2014;. doi:10.3748/wjg.v20.i7.1635.

41. An JY, Youn HG, Choi MG, Noh JH, Sohn TS, Kim S. The difficult choice between total and proximal gastrectomy in proximal early gastric cancer. Am J Surg. 2008;. doi:10.1016/j. amjsurg.2007.09.040.

42. Maruyama K, Sasako M, Kinoshita T, Sano T, Katai H. Surgical treatment for gastric cancer: the Japanese approach. Semin Oncol. 1996;23:360-8.

43. Karimi P, Islami F, Anandasabapathy S, Freedman ND, Kamangar F. Gastric cancer: descriptive epidemiology, risk factors, screening, and prevention. Cancer Epidemiol Biomark Prev. 2014;. doi:10.1158/1055-9965.EPI-13-1057.

44. Kamangar F, Sheikhattari P, Mohebtash M. Helicobacter pylori and its effects on human health and disease. Arch Iran Med. 2011. doi: 011143/AIM.0010. 
45. Lagergren J, Bergström R, Lindgren A, Nyrén O. Symptomatic gastroesophageal reflux as a risk factor for esophageal adenocarcinoma. N Engl J Med. 1999;340:825-31.

46. Derakhshan MH, Malekzadeh R, Watabe H, Yazdanbod A, Fyfe $\mathrm{V}$, Kazemi A, et al. Combination of gastric atrophy, reflux symptoms and histological subtype indicates two distinct aetiologies of gastric cardia cancer. Gut. 2008;57:298-305.

47. Pinheiro PS, Morris CR, Liu L, Bungum TJ, Altekruse SF. The impact of follow-up type and missed deaths on population-based cancer survival studies for Hispanics and Asians. J Natl Cancer Inst Monogr. 2014;. doi:10.1093/jncimonographs/lgu016.

48. Razum O. Commentary: of salmon and time travelers-musing on the mystery of migrant mortality. Int $\mathrm{J}$ Epidemiol. 2006;35:919-21.
49. Acciai F, Noah AJ, Firebaugh G. Pinpointing the sources of the Asian mortality advantage in the USA. J Epidemiol Community Health. 2015; . doi:10.1136/jech-2015-205623.

50. Tendulkar SA, Hamilton RC, Chu C, Arsenault L, Duffy K, Huynh V, et al. Investigating the myth of the "model minority": a participatory community health assessment of Chinese and Vietnamese adults. J Immigr Minor Health. 2012;. doi:10.1007/ s10903-011-9517-y.

51. Glenn BA, Chawla N, Surani Z, Bastani R. Rates and sociodemographic correlates of cancer screening among South Asians. J Community Health. 2009; doi:10.1007/s10900-008-9129-1.

52. Hwang H. Colorectal cancer screening among Asian Americans. Asian Pac J Cancer Prev. 2013;14:4025-32. 